\title{
Equation of motion approach to non-adiabatic quantum charge pumping
}

\author{
Amit Agarwal and Diptiman Sen \\ Centre for High Energy Physics, Indian Institute of Science, Bangalore 560012, India
}

(July 14, 2018)

\begin{abstract}
We use the equations of motion of non-interacting electrons in a one-dimensional system to numerically study different aspects of charge pumping. We study the effects of the pumping frequency, amplitude, band filling and finite bias on the charge pumped per cycle, and the Fourier transforms of the charge and energy currents in the leads. Our method works for all values of parameters, and gives the complete time-dependences of the current and charge at any site of the system. Our results agree with Floquet and adiabatic scattering theory where these are applicable, and provides support for a mechanism proposed elsewhere for charge pumping by a traveling potential wave. For non-adiabatic and strong pumping, the charge and energy currents are found to have a marked asymmetry between the two leads, and pumping can work even against a substantial bias.
\end{abstract}

PACS number: 73.23.-b, 72.10.Bg, 73.63.Nm

\section{INTRODUCTION}

The idea that periodically oscillating potentials applied at certain sites of a system can transfer a net charge per cycle between two leads (which are at the same chemical potential) has been studied for several years, both theoretically [1-18] and experimentally $[19,20]$. Theoretical studies have used adiabatic scattering theory [5,6], Floquet scattering theory $[8,9]$ and variations of the non-equilibrium Green function (NEGF) formalism [10-12]. While Floquet scattering theory and the NEGF formalism work for potentials oscillating with any frequency, adiabatic theory works only for low frequencies. All these methods provide expressions for the charge transferred per cycle. However, it is not easy to obtain from these methods the current and charge at any site as a function of time. Detailed information like this may shed light on the mechanism of charge pumping, for instance, by a traveling potential wave which has been observed in several experiments [20]. Further, the effects of a finite bias between the leads are not easy to study analytically unless the pumping is adiabatic [21]. In this paper, we present a numerical method for obtaining all this information for a system of non-interacting electrons. Our method is based on solving the equation of motion (EOM) of the density matrix of the system [22-24]. (For time-independent Hamiltonians, this method reproduces the results obtained by the NEGF formalism [25]). We will see that the time-dependences of the currents and charges depend significantly on the amplitude and frequency of the pumping. We will show that there is a marked asymmetry in the Fourier transforms of the charge and heat currents in the two leads if the pumping amplitude and frequency are large. We will also show that pumping can work even if there is a substantial bias opposing it.

As a simple model for studying charge pumping, we consider a one-dimensional system consisting of two semiinfinite leads $a=L, R$ (denoting left and right) and a finite region $W$ (wire) lying between the two. We will model all three regions by lattices with electrons governed by a one-channel tight-binding Hamiltonian with the same hopping amplitude $-\gamma$ on all bonds, namely,

$$
\hat{H}_{0}=-\gamma \sum_{n=1}^{N-1}\left(c_{n+1}^{\dagger} c_{n}+c_{n}^{\dagger} c_{n+1}\right),
$$

where $N$ is the total number of sites. We will consider spinless electrons here; the current of spin- $1 / 2$ electrons is simply twice that of spinless electrons for non-interacting electrons. The dispersion of the electrons in the leads is $E_{k}=-2 \gamma \cos k$, where $k$ lies in the range $[-\pi, \pi]$. (We are setting the Planck constant $\hbar$ and the lattice spacing equal to unity). The two leads are assumed to have the same chemical potential $\mu$ and temperature $T$. Time-dependent potentials will be applied to some sites of the wire; that part of the Hamiltonian is given by

$$
\hat{V}(t)=\sum_{n} V_{n}(t) c_{n}^{\dagger} c_{n}, \quad \text { where } V_{n}(t)=a_{n} \cos \left(\omega t+\phi_{n}\right) .
$$

The sites with these potentials will be collectively called the scattering region. 
In Secs. II and III, we will describe how Floquet scattering theory and adiabatic scattering theory respectively can be used to study charge pumping in the above model. In Sec. IV, we will describe the EOM method for numerically studying various quantities of interest. Numerical results will be presented in Sec. V. In Sec. VI, we will summarize our results and point out some problems for future studies.

\section{FLOQUET SCATTERING THEORY}

Briefly, Floquet scattering theory works as follows $[8,9]$. The incoming electrons of energy $E_{0}$ gain or lose energy in quanta of $\omega$ on interacting with the scattering region. Hence, the outgoing states are characterized by energies $E_{p}=E_{0}+p \omega$, where $p=0, \pm 1, \pm 2, \cdots ;$ the energies with $p \neq 0$ are called the Floquet side bands. The effect of the scattering region can be described by a Floquet scattering matrix $S_{\alpha \beta}\left(E_{p}, E_{0}\right)$, which is the amplitude for an electron with energy $E_{0}$ entering through lead $\beta$ to leave with energy $E_{p}$ through lead $\alpha$. In the leads, the propagating modes have energies lying within the bandwidth $[-2 \gamma, 2 \gamma]$; only these modes can contribute to charge pumping. States with energies lying outside the bandwidth have wave functions which decay exponentially into the leads and hence do not contribute to charge transfer. The wave function of an electron coming from the left lead with an energy $E_{0}$ and wavenumber $k_{0}$ (with $E_{0}=-2 \gamma \cos k_{0}$ ) is given by

$$
\psi(n)=e^{i\left(k_{0} n-E_{0} t\right)}+\sum_{p} r_{p} e^{i\left(-k_{p} n-E_{p} t\right)},
$$

at a site $n$ far to the left of the scattering region, and

$$
\psi(n)=\sum_{p} t_{p} e^{i\left(k_{p} n-E_{p} t\right)}
$$

far to the right of the scattering region, where $E_{p}=-2 \gamma \cos k_{p}$, and the sums over $p$ run over values such that $E_{p}$ lies within the bandwidth of the leads. The quantities $r_{p}$ and $t_{p}$ denote reflection and transmission amplitudes in the different side bands; they respectively denote the elements $S_{L L}\left(E_{p}, E_{0}\right)$ and $S_{R L}\left(E_{p}, E_{0}\right)$ of the Floquet scattering matrix, where $L$ and $R$ denote the left and right leads. Similarly, the wave function of an electron coming from the right lead with an energy $E_{0}$ and wavenumber $k_{0}$ is given by

$$
\psi(n)=e^{i\left(-k_{0} n-E_{0} t\right)}+\sum_{p} \bar{r}_{p} e^{i\left(k_{p} n-E_{p} t\right)},
$$

far to the right of the scattering region, and

$$
\psi(n)=\sum_{p} \bar{t}_{p} e^{i\left(-k_{p} n-E_{p} t\right)},
$$

far to the left of the scattering region. The reflection and transmission amplitudes are found by writing down the wave functions in the scattering region, and matching coefficients of terms having the same time dependence $\left(e^{ \pm i E_{p} t}\right)$ in the Schrödinger equation at different sites. If the oscillating potentials are weak, the reflection and transmission amplitudes decrease rapidly as $|p|$ increases; at first order in the potentials, only $p= \pm 1$ contribute. The current in, say, the right lead is then given by

$$
\begin{aligned}
I_{R}=q \int_{-2 \gamma}^{2 \gamma} \frac{d E_{0}}{2 \pi}[ & \frac{v_{1}}{v_{0}}\left(\left|t_{1}\right|^{2}+\left|\bar{r}_{1}\right|^{2}\right)\left\{f\left(E_{0}, \mu, T\right)-f\left(E_{1}, \mu, T\right)\right\} \\
& +\frac{v_{-1}}{v_{0}}\left(\left|t_{-1}\right|^{2}+\left|\bar{r}_{-1}\right|^{2}\right)\left\{\left(f\left(E_{0}, \mu, T\right)-f\left(E_{-1}, \mu, T\right)\right\}\right],
\end{aligned}
$$

where $f(E, \mu, T)=1 /\left[e^{(E-\mu) / k_{B} T}+1\right]$ is the Fermi function, and $v_{p}=2 \gamma \sin k_{p}$ is the velocity. In the limit $\omega \rightarrow 0$, we have $f\left(E_{ \pm 1}, \mu, T\right)-f\left(E_{0}, \mu, T\right)= \pm \omega \partial f\left(E_{0}, \mu, T\right) / \partial E_{0}$, and $v_{p} / v_{0} \rightarrow 1$. Finally, at zero temperature, $\partial f\left(E_{0}, \mu, 0\right) / \partial E_{0}=-\delta\left(E_{0}-\mu\right)$.

\section{ADIABATIC SCATTERING THEORY}

In the limit of the pumping frequency tending to zero, the charge transport can be related to the 'frozen' scattering matrix $S[2,3,5]$. The average pumped current in this limit is found to be proportional to the frequency $\omega$, and 
therefore the charge pumped per cycle (of time period $2 \pi / \omega$ ) is independent of $\omega$. If the scattering region is connected to leads which are at the same chemical potential and zero temperature, the infinitesimal charge flowing from that region to the $a$-th lead is given by

$$
d Q_{a}=\frac{i q}{2 \pi}\left(d S S^{\dagger}\right)_{a a}
$$

where $q$ is the electron charge, and the 'frozen' $S$-matrix is evaluated at the Fermi energy $E_{F}=-2 \gamma \cos k_{F}=\mu$. Eq. (8) can be used to relate adiabatic scattering theory to a geometric description of charge transport [3]. For weak oscillating potentials $V_{n}$ as given in Eq. (2), one finds that the charge entering lead $a$ per cycle is given by

$$
\Delta Q_{a}=-\frac{q}{\pi} \sum_{n>m} \operatorname{Im}\left[\left(\frac{\partial S}{\partial V_{n}} \frac{\partial S^{\dagger}}{\partial V_{m}}\right)_{\left\{V_{m}\right\}=0}\right]_{a a} \oint d V_{n} V_{m}
$$

where the integral is done over one cycle of the oscillation. Eq. (9) is a generalization of the formula in Ref. [3] and can be derived from Eq. (8) by Taylor expanding the scattering matrix to first order, $S\left(V_{m}\right)=S(0)+$ $\sum_{n} V_{n}\left(\partial S / \partial V_{n}\right)_{\left\{V_{m}\right\}=0}$. In the case of two weak oscillating potentials $V_{1}(t)$ and $V_{2}(t)$, Eq. $(9)$ shows that the pumped charge is proportional to the area in the space of the parameters $\left(V_{1}, V_{2}\right)$.

\section{EQUATION OF MOTION METHOD}

For a system with a finite number of sites, we study the time evolution as follows. The density matrix of the system evolves according to the EOM

$$
\hat{\rho}(t+d t)=e^{-i \hat{H}(t) d t} \hat{\rho}(t) e^{i \hat{H}(t) d t},
$$

where $\hat{H}(t)=\hat{H}_{0}+\hat{V}(t)$ is given in Eqs. (1-2). The current across any bond is then obtained by taking the trace of the appropriate current operator with $\hat{\rho}$. The current operator on the bond from site $n$ to site $n+1$ and its expectation value at time $t$ are given by

$$
\begin{aligned}
\hat{j}_{n+1 / 2} & =i q \gamma\left(c_{n+1}^{\dagger} c_{n}-c_{n}^{\dagger} c_{n+1}\right) \\
\text { and } \quad j_{n+1 / 2}(t) & =\operatorname{Tr}\left(\hat{\rho}(t) \hat{j}_{n+1 / 2}\right)=i q \gamma\left[\hat{\rho}_{n, n+1}(t)-\hat{\rho}_{n+1, n}(t)\right] .
\end{aligned}
$$

The charge transferred between the right and left leads $R$ and $L$ between two times can be found either by integrating the above expression in time, or by taking the operator

$$
\Delta \hat{Q}=\frac{q}{2}\left[\sum_{n \in R} c_{n}^{\dagger} c_{n}-\sum_{n \in L} c_{n}^{\dagger} c_{n}\right]
$$

and computing $\operatorname{Tr}(\hat{\rho}(t) \Delta \hat{Q})$ at the two times; these methods give the same result for the charge transferred in a cycle. Similarly, one can compute the energy current across any site $n$; the corresponding operator and its expectation value are given by

$$
\begin{aligned}
\hat{e}_{n} & =-i \gamma^{2}\left(c_{n+1}^{\dagger} c_{n-1}-c_{n-1}^{\dagger} c_{n+1}\right), \\
\text { and } \quad e_{n}(t) & =\operatorname{Tr}\left(\hat{\rho}(t) \hat{e}_{n}\right)=-i \gamma^{2}\left[\hat{\rho}_{n-1, n+1}(t)-\hat{\rho}_{n+1, n-1}(t)\right],
\end{aligned}
$$

in a region where the on-site potential $V_{n}$ is zero [22]. At zero temperature, the charge current is carried by electrons whose average energy is given by $E_{F}=\mu$; the difference between the energy current $e$ and the charge current $j$ multiplied by $\mu$ gives the heat current $[5,8]$. We define the heat current at site $n$ to be $e_{n}-(\mu / 2)\left(j_{n-1 / 2}+j_{n+1 / 2}\right)$.

In all our calculations, we take the left and right leads to have $N_{L}$ sites each and the wire in the middle to have $N_{W}$ sites; the total number of sites is $N=2 N_{L}+N_{W}$. We set the hopping amplitude $\gamma=1$. For calculations in which the chemical potential is the same in the two leads, we take the density matrix at time $t=0$ to be given by that of a single system governed by the Hamiltonian $H_{0}$ in Eq. (1) with $N$ sites, chemical potential $\mu$ and temperature $T$ (which we will take to be zero). If $E_{\alpha}$ and $\psi_{\alpha}(n)$ are the eigenvalues and eigenstates of the $\hat{H}_{0}(\alpha$ and $n$ label the states and sites respectively), the initial density matrix is given by 


$$
\hat{\rho}_{m n}(0)=\sum_{\alpha} \psi_{\alpha}(m) \psi_{\alpha}^{*}(n) f\left(E_{\alpha}, \mu, T\right) .
$$

We then evolve the density matrix in time and compute the current and charge transferred using Eqs. (10-12).

We will also be interested in calculations in which the chemical potentials are not the same in the two leads; suppose that they are given by $\mu_{L}$ and $\mu_{R}$ in the left and right leads respectively. In this case, we begin by setting the hopping amplitude to be zero on one of the bonds in the middle of the system; we then compute the density matrices $\hat{\rho}_{L}$ and $\hat{\rho}_{R}$ in the left and right parts of the system by restricting Eq. (14) to the left and right leads respectively. The complete density matrix $\hat{\rho}(0)$ is then given by the direct sum of $\hat{\rho}_{L}$ and $\hat{\rho}_{R}$. We then set the hopping amplitude on that bond to unity, and evolve the density matrix with the Hamiltonian $\hat{H}(t)$ as usual.

We should note that although the net charge transferred per cycle is the same across all bonds, the detailed time dependence of the charge transferred looks different for different bonds. (This will become particularly clear in a later figure). Another point to note is that the finite length of the leads (with $N_{L}$ sites) implies that the system has a return time $T_{R}$ equal to $2 N_{L} / v_{F}$ where the Fermi velocity $v_{F}=2 \gamma \sin k_{F}$ [23]; this is the time required for an electron to travel from the wire in the middle to the end of either of the two leads and then return to the wire. The numerical results can be trusted only for times which are less than $T_{R}$. Finally, there are transient effects which last for one or two cycles; the effects of different choices of the initial density matrix get washed out after this transient period. All the numerical results presented below are therefore taken from times which are larger than the transient time but smaller than $T_{R}$.

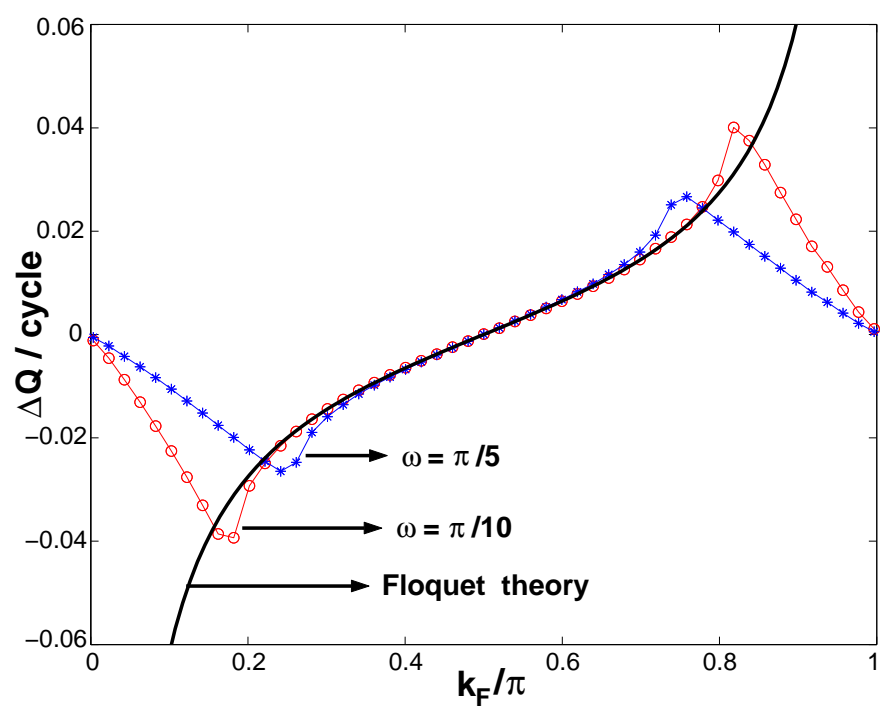

FIG. 1. Charge transferred (in units of $q$ ) per cycle from left to right versus the Fermi wavenumber for an oscillating potential at 2 sites, with $a_{1}=a_{2}=0.2, \phi_{2}-\phi_{1}=\pi / 2$, and $\omega=\pi / 10$ (circles) and $\pi / 5$ (stars) for a system with 202 sites $\left(N_{L}=100\right)$. The solid line shows the results obtained using second order Floquet scattering theory in the limit $\omega \rightarrow 0$.

\section{NUMERICAL RESULTS}

\section{A. Effect of band filling}

Figure 1 shows the charge transferred versus the Fermi wavenumber $k_{F}$ as obtained by the EOM method when oscillating potentials are only applied at two neighboring sites. If the amplitudes $a_{1}$ and $a_{2}$ are much smaller than $v_{F}$, one can use Floquet scattering theory to second order in the $a_{n}$ to find the charge transferred per cycle from left to right in the limit $\omega \rightarrow 0$,

$$
\Delta Q=-q \frac{a_{1} a_{2}}{\gamma^{2}} \sin \left(\phi_{2}-\phi_{1}\right) \frac{\cot k_{F}}{2} .
$$

The comparison between the results obtained numerically for two different pumping frequencies and the analytical expression given in Eq. (15) is also shown in Fig. 1. We see that the numerical results and second order Floquet 
scattering theory match near the middle of the band, $k_{F}=\pi / 2$, but the agreement becomes poor near the band edges $k_{F}=0$ and $\pi$. This discrepancy is due to the expansion parameter $a_{n} / v_{F}$ becoming large near the band edges; hence second order perturbation theory breaks down, and Eq. (15) is no longer valid. We can also define an adiabaticity parameter $\epsilon=\left(d / v_{F}\right) /(2 \pi / \omega)$ which is the ratio of the time taken for an electron with Fermi velocity $v_{F}$ to traverse the scattering region of length $d$ to the time period of the oscillating potentials; the adiabatic limit corresponds to $\epsilon \rightarrow 0$. In our case, $d=1$ (the oscillating potentials are at neighboring sites), while $v_{F}=2$ at $k_{F}=\pi / 2$ (half-filling). Even for the largest frequency of $\omega=\pi / 5$ used in Fig. 1, we see that $\epsilon=1 / 20$. This is why the two curves with finite $\omega$ collapse on to the results of Floquet scattering theory near $k_{F}=\pi / 2$; this may not happen if $\epsilon$ is of order 1 , i.e., if either $\omega$ or $d$ becomes larger.

Fig. 1 shows that the charge transferred goes through an extremum as $k_{F}$ approaches the band edges $(0$ or $\pi)$. This can be understood as follows. For a weak potential $\left(a_{1}, a_{2}\right.$ small), only the first Floquet side bands $(p= \pm 1)$ contribute to the current. From the structure given in Eq. (7), one can then see that at zero temperature, the current gets a contribution only from electrons which lie within the energy range $\left[E_{F}-\omega, E_{F}\right]$ initially and get excited to the energy range $\left[E_{F}, E_{F}+\omega\right]$ finally. The number of states lying within these ranges starts decreasing when one gets very close to the band edges, namely, when $E_{F}-\omega$ goes below the bottom end of the band, or $E_{F}+\omega$ goes above the top end. (We recall that the band has a finite width going from $-2 \gamma$ to $2 \gamma$ ). Hence the magnitude of the current starts decreasing when $E_{F}-\omega$ falls below $-2 \gamma$ or when $E_{F}+\omega$ goes above $2 \gamma$. Qualitatively, this is what one observes in Fig. 1; the charge transferred goes through an extremum when $E_{F}=-2 \gamma k_{F}$ gets within a distance of $\omega$ from the top end or the bottom end of the band. The extremum occurs closer to the band edge if $\omega$ is smaller $(\pi / 10$ instead of $\pi / 5)$.

Note that the Hamiltonian in Eqs. (1-2) is invariant under the particle-hole transformation $c_{n} \rightarrow(-1)^{n} c_{n}^{\dagger}$ and $t \rightarrow t+\pi / \omega$, but the current operator changes sign. Hence the charge transferred is antisymmetric about $k_{F}=\pi / 2$ (half-filling) as we can see in Fig. 1; hence the pumped charge is exactly zero at half-filling for any value of the frequency $\omega$.

\section{B. Traveling potential wave}

Eq. (15) can be generalized to the case in which there are oscillatory potentials at several sites as given in Eq. (2). In the limit $\omega \rightarrow 0$, the use of Floquet scattering theory up to second order in the amplitudes gives the charge transferred per cycle from left to right to be

$$
\Delta Q=-q \sum_{n>m} \frac{a_{n} a_{m}}{\gamma^{2}} \sin \left(\phi_{n}-\phi_{m}\right) \frac{\sin \left[2 k_{F}(n-m)\right]}{4 \sin ^{2} k_{F}} .
$$

Since the amplitudes $a_{n}$ are positive, Eq. (16) suggests that the charge transferred will be maximized if one chooses $\phi_{n}-\phi_{m}$ to be in phase with $2 k_{F}(n-m)$ for all pairs of sites $n$ and $m$. A simple way to ensure this is to choose $\phi_{n}=2 k_{F} n$. This is the choice of the phases $\phi_{n}$ made in Figs. 2-6 all of which involve systems with oscillating potentials applied to 8 consecutive sites, with the same amplitude $a$ at all those sites. Hence the potential at site $n$ takes the form $a \cos \left(\omega t+2 k_{F} n\right)$; this describes a potential wave traveling with a velocity $\omega / 2 k_{F}$.

Figures 2 and 3 compare the cases of weak and strong pumping. The Fourier transform of the current (computed at the ninth bond to the right of the scattering region) shows that for weak pumping $(a=0.2)$, only a small number of Floquet side bands contribute to the current, while for strong pumping $(a=2)$, a large number of Floquet side bands contribute. The Fourier transform of the current therefore provides a way of distinguishing between strong and weak pumping.

As an application of the EOM method to recent observations of charge pumping by a traveling potential wave $[15,20]$, we present in Fig. 4 the charge transferred versus time in one cycle in the strong pumping regime, as obtained by the EOM method for $\omega=\pi / 100$ and $\pi / 200$, and from Eq. (8) for the adiabatic case. We see that very little charge is transferred in one part of the cycle, and a lot of charge is transferred in the other part; the reason for this will become clear below. The charge transferred per cycle is about 1.4 for $\omega=\pi / 100,1.8$ for $\omega=\pi / 200$, and exactly 2 in the adiabatic case. In this model, therefore, the charge transferred increases as the pumping becomes more adiabatic.

Figure 5 shows the density profile, $\operatorname{Tr}\left(\hat{\rho} c_{n}^{\dagger} c_{n}\right)$, in and on two sides of the scattering region at eight equally spaced times in one cycle for the same parameters as in Fig. 4, with $\omega=\pi / 200$. We see a larger number of electrons in the regions where the potential has a minimum; these electrons move along with the potential minimum. The first six pictures in Fig. 5 show some electrons (about 1.8 in number as indicated in Fig. 4) being transported by the potential minimum from the right side of the scattering region to the left; during this period, very little charge is transferred to or from the leads. The last two pictures in Fig. 5 show these electrons being transmitted to the left 


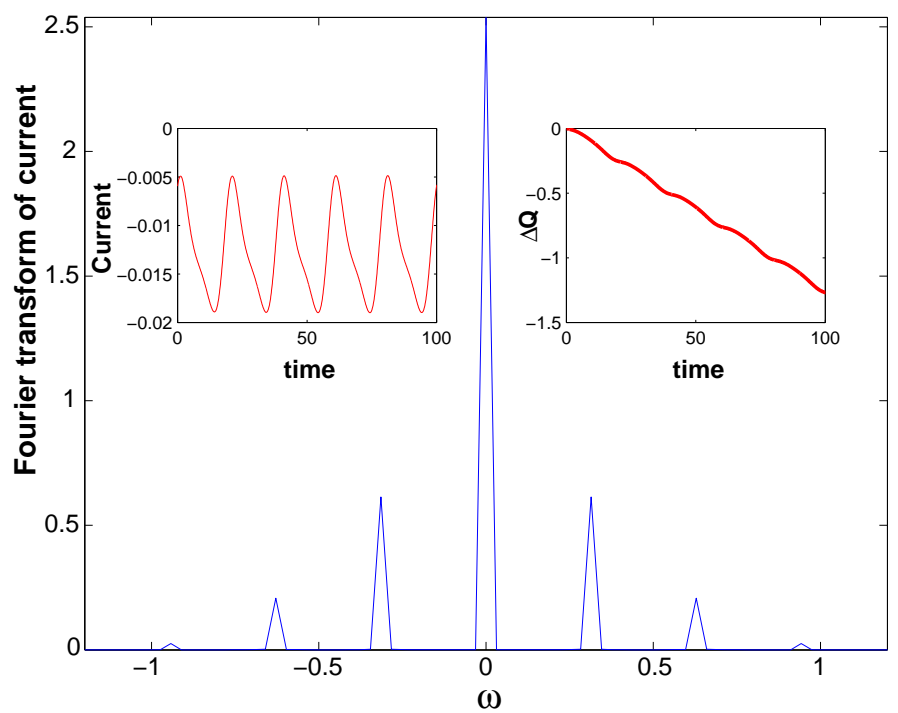

FIG. 2. Fourier transform of the current (in units of $q$ ) versus frequency for oscillating potentials at 8 sites, with $a=0.2$, $\omega=\pi / 10$ and $k_{F}=\pi / 4$ for a system with 638 sites $\left(N_{L}=315\right)$. The left and right insets show the current and charge transferred versus time.

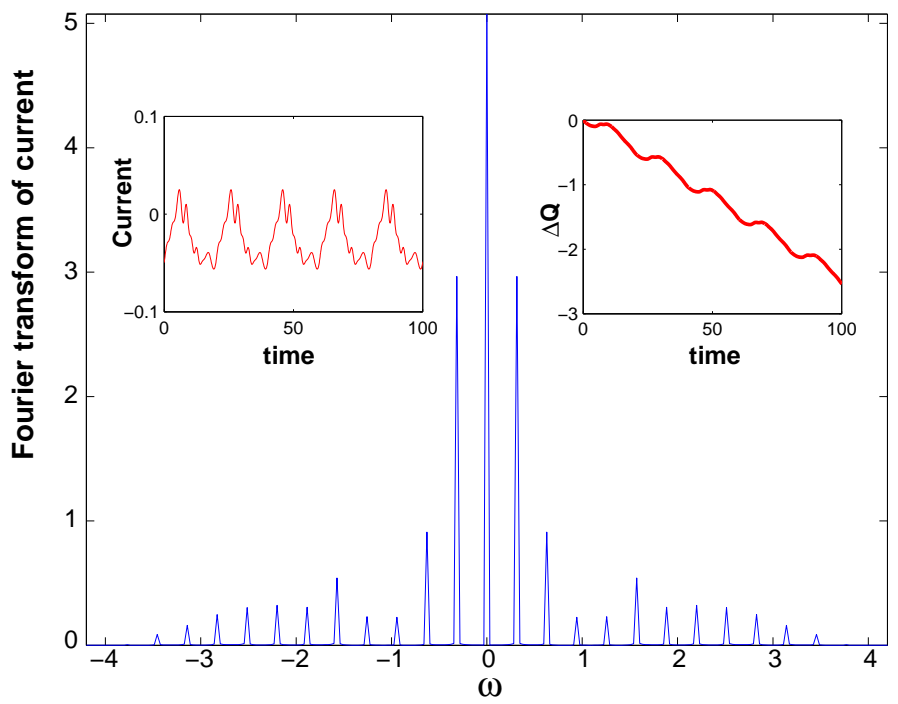

FIG. 3. Fourier transform of the current (in units of $q$ ) versus frequency for oscillating potentials at 8 sites, with $a=2$, $\omega=\pi / 10$ and $k_{F}=\pi / 4$ for a system with 638 sites $\left(N_{L}=315\right)$. The left and right insets show the current and charge transferred versus time.

lead, while some other electrons are entering the scattering region from the right lead. These pictures illustrate the mechanism of charge transfer mentioned in Ref. [20]. Note that charge gets pumped in this model even though the 'frozen' $S$-matrix is almost perfectly reflecting at all times; the current would have been very small if the potential wave had been stationary.

\section{Asymmetry in charge and energy currents}

Although the time-averaged current must be the same at all sites due to current conservation, the fluctuations in the current need not be the same everywhere. This is illustrated in Figs. 6 and 7 which show the currents at the tenth 


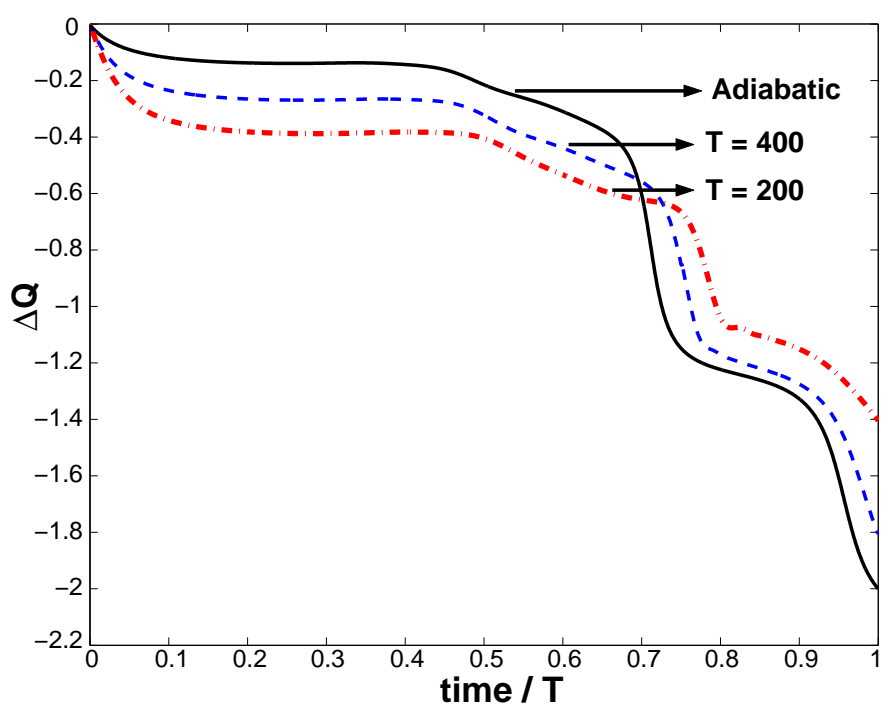

FIG. 4. Charge transferred (in units of $q$ ) from left to right versus time during one cycle (with period $T=2 \pi / \omega$ ) for oscillating potentials at 8 sites, with $a=2, k_{F}=\pi / 8$, and $\omega=\pi / 100$ (dash dot), $\pi / 200$ (dashed), and tending to zero (solid line).
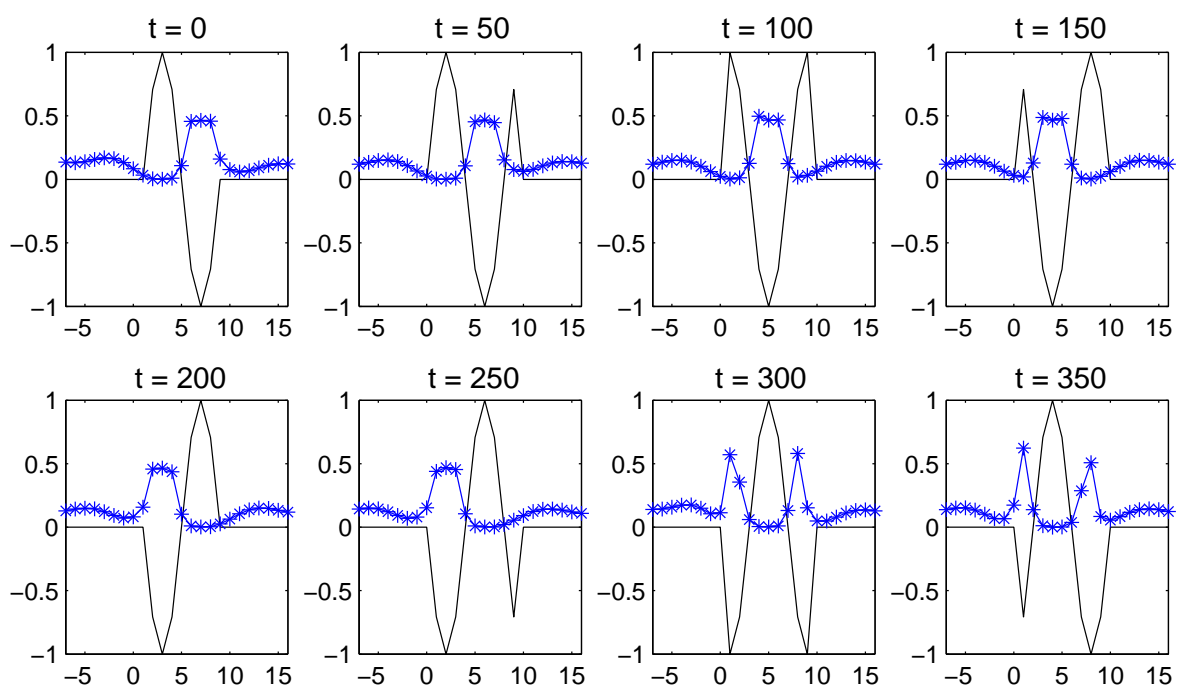

FIG. 5. Electron numbers (stars) and potentials at 24 sites in and on two sides of the scattering region (sites 1 to 8 ) at 8 equally spaced times in one cycle, for potentials oscillating with $a=2, \omega=\pi / 200$ and $k_{F}=\pi / 8$ for a system with 1618 sites $\left(N_{L}=805\right)$. The scale on the $y$-axis indicates both the electron number and the potential (divided by 2$)$ at each site.

bond on the left and tenth bond on the right respectively of the scattering region for a model with the same parameters as in Fig. 3. We see that the current is distinctly more noisy on the left. This is an effect of non-adiabaticity; we find that it disappears in the limit $\omega \rightarrow 0$. Qualitatively, this asymmetry occurs because the potential minimum 'picks' up electrons from the right lead, transports them through the scattering region with a finite velocity given by $\omega / 2 k_{F}$, and finally 'throws' them into the left lead with that velocity; the finiteness of this velocity may be responsible for the additional noise on the left.

The asymmetry can also be seen in the energy and heat currents $[5,8,22]$ on the left and right of the scattering region for the same model. Figure 8 shows the outgoing energy currents $e_{L}$ and $e_{R}$ on the tenth site on the left and tenth site on the right respectively of the scattering region, and the product of the chemical potential $\mu$ with the outgoing charge currents $j_{L}$ and $j_{R}$ on the left and right, all as functions of the pumping frequency $\omega$. (Due to current conservation in the steady state, $j_{L}=-j_{R}$ ). The differences between the two currents, namely, $e_{L}-\mu j_{L}$ and 


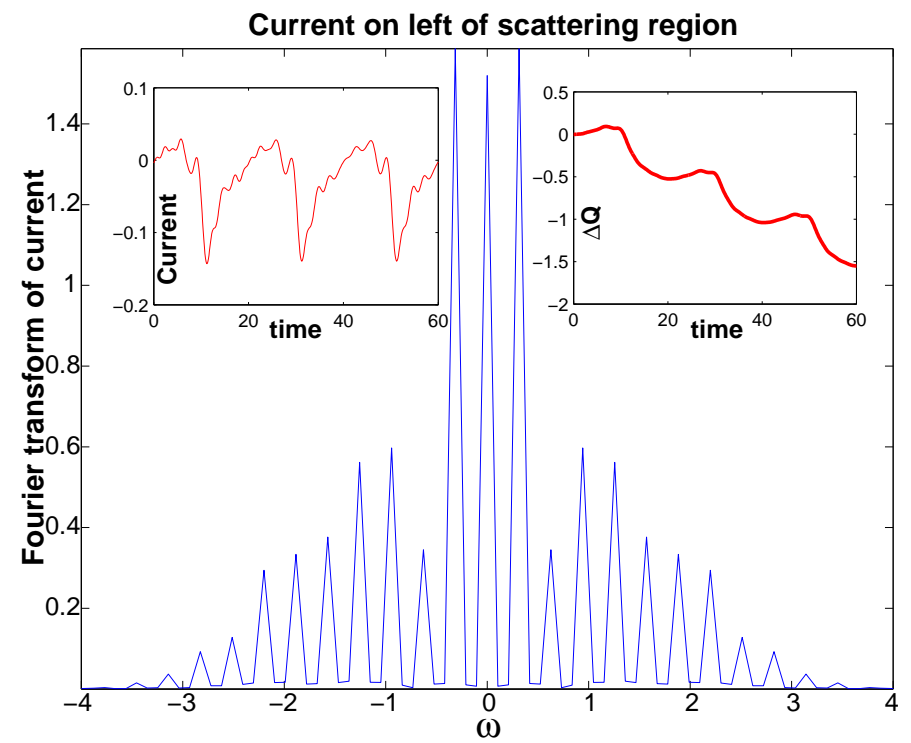

FIG. 6. Fourier transform of the current (in units of $q$ ) on the left of the scattering region versus frequency for oscillating potentials at 8 sites, with $a=2, \omega=\pi / 10$ and $k_{F}=\pi / 4$ for a system with 428 sites $\left(N_{L}=210\right)$. The left and right insets show the current and charge transferred versus time.

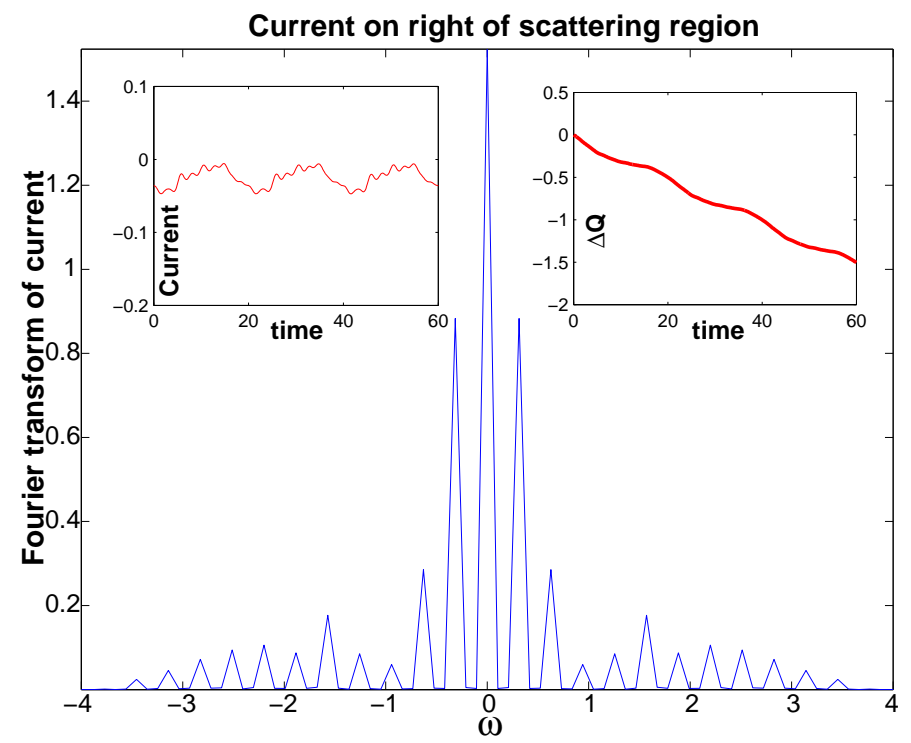

FIG. 7. Fourier transform of the current (in units of $q$ ) on the right of the scattering region versus frequency for oscillating potentials at 8 sites, with $a=2, \omega=\pi / 10$ and $k_{F}=\pi / 4$ for a system with 428 sites $\left(N_{L}=210\right)$. The left and right insets show the current and charge transferred versus time.

$e_{R}-\mu j_{R}$ give the heat currents on the left and right; these are shown in the inset of Fig. 8. We see that the heat current on the left is significantly larger than on the right. We have not attempted to determine quantitatively how the heat current varies with $\omega$; the dependence is known to be quadratic for small $\omega[5,8]$.

We should emphasize here that our model has no mechanisms (such as electron-phonon scattering) for heat dissipation in the leads. A real system will have such mechanisms, and the heat currents calculated above will eventually get dissipated somewhere in the leads. 


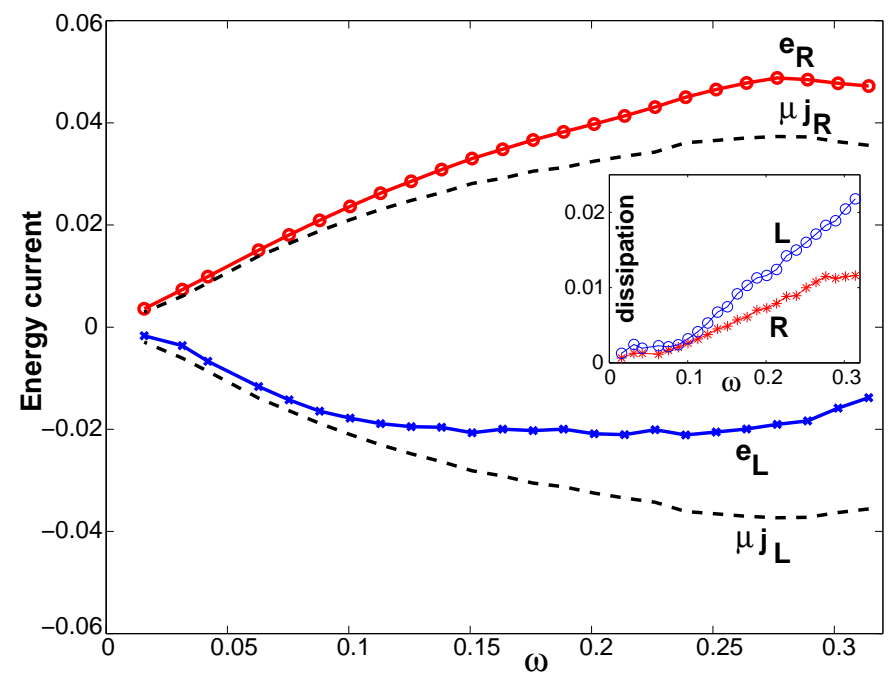

FIG. 8. Energy currents $e_{L}$ and $e_{R}$ on the left and right of the scattering region versus frequency for oscillating potentials at 8 sites, with $a=2$ and $k_{F}=\pi / 4$. The curves marked as $\mu j_{L}$ and $\mu_{R}$ show the product of the chemical potential with the charge currents $j_{L}$ and $j_{R}$ on the left and right. The inset shows the heat current on the left and right versus frequency; these are given by $e_{L}-\mu j_{L}$ and $e_{R}-\mu j_{R}$ respectively.

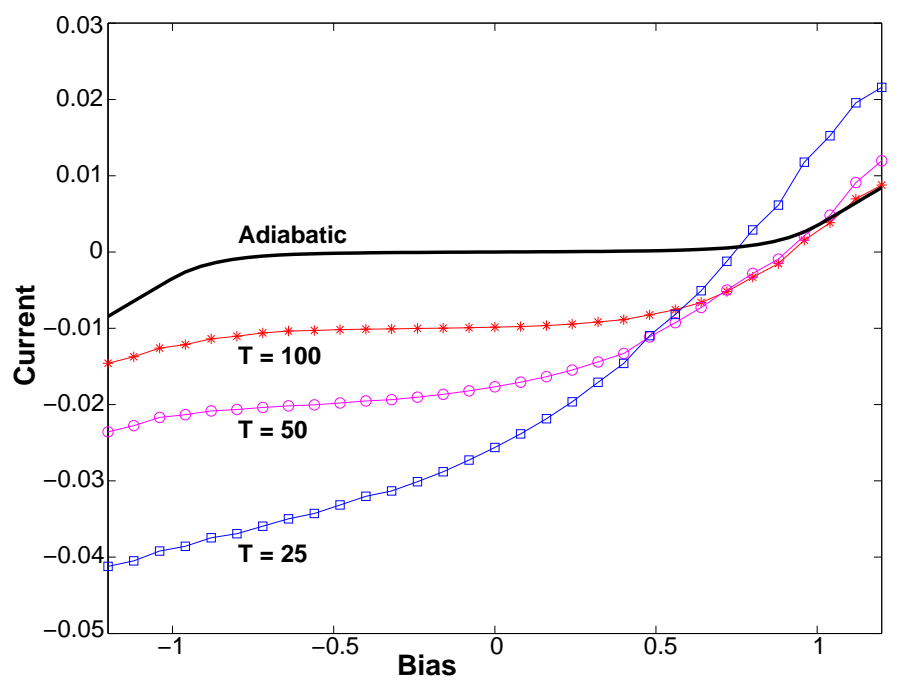

FIG. 9. Current (in units of $q$ ) versus bias for different frequencies (with $T=2 \pi / \omega$ ) for oscillating potentials at 8 sites, with $a=2$ and $k_{F}=\pi / 4$ for a system with 428 sites $\left(N_{L}=210\right)$.

\section{Effect of finite bias}

We will now consider the effect of a finite bias on the current [21]. We take the chemical potential in the left and right leads to be $\mu_{L}=\mu+q V / 2$ and $\mu_{R}=\mu-q V / 2$ respectively, so that there is a bias of $q V$ between left and right. The calculations are done as outlined in Sec. IV. Fig. 9 shows the effect of a bias on the current for different frequencies for the same model as in Fig. 3. As expected, the current from left to right increases with the bias; however, the current continues to be negative (i.e., flows from right to left) for a range of positive values of the bias. For a pumping frequency of $\omega=2 \pi / 100 \simeq 0.063$, charge can get pumped against an opposing bias of almost 0.9 . We also observe a pronounced asymmetry between positive and negative values of the bias, and the asymmetry increases with the pumping frequency. Finally, note that the current is very small for small bias in the adiabatic limit; this is because the scattering region is almost perfectly reflecting, as was mentioned earlier. In the adiabatic limit, we have 
computed the current analytically using the Landauer-Büttiker expression averaged over time [21]. Namely,

$$
I_{R}=q \int_{-2 \gamma}^{2 \gamma} \frac{d E}{2 \pi}<\left|S_{R L}(E, t)\right|^{2}>_{t}[f(E, \mu+q V / 2, T)-f(E, \mu-q V / 2, T)],
$$

where $<\left|S_{R L}(E, t)\right|^{2}>_{t}$ denotes the average over one time period of the 'frozen' transmission probability from left to right for a given energy $E$.

\section{DISCUSSION}

The EOM method provides a general way of computing the charge, energy and heat currents as functions of time at different sites. A knowledge of the detailed space-time dependence of currents and charges is often useful. For instance, our results give an insight into the mechanism of charge pumping by a traveling potential wave which has been studied experimentally in several systems [20]. Namely, there are more electrons in a region in which the potential is attractive; as this region moves with time, so do those electrons. In this way, electrons are transported across the wire from one lead to another. We find that non-adiabatic charge pumping by a traveling potential wave produces an appreciable asymmetry in the Fourier transforms of the charge and heat currents. It would be interesting to experimentally look for an asymmetry in the Fourier transform of the current in the different systems where charge pumping has been demonstrated.

The EOM approach is quite versatile and makes no assumptions about the ranges of the different parameter values; the potentials and pumping frequency may be small or large, and the potentials may vary with time in an arbitrary way, not necessarily simple harmonic or even periodic. The EOM method can also be used to study pumping at resonant frequencies $[8,17]$.

It may be useful to compare the EOM and NEGF methods here. The EOM method requires long leads in order to have a large return time, so that one has a reasonable window of time to compute various quantities of interest. It can be extended to the case of two- and three-dimensional leads [24]; however the calculations involve significantly larger systems in those cases. The NEGF formalism involves a self-energy $\Sigma(E)$ which takes the leads into account in an exact way; hence large leads do not need to be explicitly included in the numerical computations. On the other hand, for time-dependent problems such as charge pumping, the NEGF formalism must work with Green functions which would generally depend on two time arguments through the self-energy $\Sigma\left(t, t^{\prime}\right)$. In contrast to this, the EOM approach is local in time, and is therefore simpler to implement numerically. One can work with the NEGF approach in the frequency domain, but that works well only if the potentials vary harmonically in time. The EOM approach would work even for potentials which vary with time in an arbitrary way.

It would be useful to extend the EOM method so as to take into account interactions between the electrons [24]. For small pumping frequencies and weak interactions, one can use an adiabatic Hartree-Fock approximation; for instance, an on-site Hubbard interaction of the form $U c_{n, \uparrow}^{\dagger} c_{n, \uparrow} c_{n, \downarrow}^{\dagger} c_{n, \downarrow}$ can be approximated by the time-dependent term $U\left[\hat{\rho}_{n, n, \uparrow}(t) c_{n, \downarrow}^{\dagger} c_{n, \downarrow}+\hat{\rho}_{n, n, \downarrow}(t) c_{n, \uparrow}^{\dagger} c_{n, \uparrow}\right]$. However, this approximation will break down if the interactions are strong, and also if the pumping frequency is large (in which case the effective on-site interaction may not instantly follow the on-site densities). We would therefore require a different technique in such situations. Recently, TomonagaLuttinger liquid theory has been used to study the effects of oscillating potentials in interacting systems [26-29].

\section{ACKNOWLEDGMENTS}

A.A. thanks CSIR, India for a Junior Research Fellowship. D.S. thanks S. Datta, A. Dhar and S. Rao for stimulating discussions. We thank DST, India for financial support under projects SR/FST/PSI-022/2000 and SP/S2/M-11/2000.

[1] D. J. Thouless, Phys. Rev. B 27, 6083 (1983); Q. Niu, Phys. Rev. Lett. 64, 1812 (1990).

[2] M. Büttiker, H. Thomas, and A. Prêtre, Z. Phys. B 94, 133 (1994).

[3] P. W. Brouwer, Phys. Rev. B 58, R10135 (1998).

[4] P. W. Brouwer, Phys. Rev. B 63, 121303(R) (2001). 
[5] J. E. Avron, A. Elgart, G. M. Graf, and L. Sadun, Phys. Rev. Lett. 87, 236601 (2001); Phys. Rev. B 62, R10618 (2000); J. Stat. Phys. 116, 425 (2004).

[6] O. Entin-Wohlman and A. Aharony, Phys. Rev. B 66, 035329 (2002).

[7] V. Kashcheyevs, A. Aharony, and O. Entin-Wohlman, Phys. Rev. B 69, 195301 (2004).

[8] M. Moskalets and M. Büttiker, Phys. Rev. B 66, 205320 (2002), and Phys. Rev. B 68, 075303 (2003).

[9] S. W. Kim, Phys. Rev. B 66, 235304 (2002).

[10] B. Wang, J. Wang, and H. Guo, Phys. Rev. B 68, 155326 (2003), and Phys. Rev. B 65, 073306 (2002).

[11] L. Arrachea, Phys. Rev. B 72, 125349 (2005), and Phys. Rev. B 72, 121306(R) (2005).

[12] L. E. F. Foa Torres, Phys. Rev. B 72, 245339 (2005).

[13] A. Banerjee, S. Das, and S. Rao, cond-mat/0307324.

[14] S. Banerjee, A. Mukherjee, S. Rao, and A. Saha, cond-mat/0608267.

[15] G. R. Aizin, G. Gumbs, and M. Pepper, Phys. Rev. B 58, 10589 (1998); G. Gumbs, G. R. Aizin, and M. Pepper, Phys. Rev. B 60, R13954 (1999); K. Flensberg, Q. Niu, and M. Pustilnik, Phys. Rev. B 60, R16291 (1999); P. A. Maksym, Phys. Rev. B 61, 4727 (2000); Y. M. Galperin, O. Entin-Wohlman, and Y. Levinson, Phys. Rev. B 63, 153309 (2001); A. M. Robinson and C. H. W. Barnes, Phys. Rev. B 63, 165418 (2001); A. Aharony and O. Entin-Wohlman, Phys. Rev. B 65, 241401(R) (2002); V. Kashcheyevs, A. Aharony, and O. Entin-Wohlman, Eur. Phys. J. B 39, 385 (2004).

[16] M. M. Mahmoodian, L. S. Braginsky, and M. V. Entin, Phys. Rev. B 74, 125317 (2006).

[17] M. Strass, P. Hänggi, and S. Kohler, Phys. Rev. Lett. 95, 130601 (2005).

[18] S. Kohler, J. Lehmann, and P. Hänggi, Phys. Rep. 406, 379 (2005).

[19] M. Switkes, C. M. Markus, K. Campman, and A. C. Gossard, Science 283, 1905 (1999).

[20] V. I. Talyanskii, J. M. Shilton, M. Pepper, C. G. Smith, C. J. B. Ford, E. H. Linfield, D. A. Ritchie, and G. A. C. Jones, Phys. Rev. B 56, 15180 (1997); J. Cunningham, V. I. Talyanskii, J. M. Shilton, M. Pepper, M. Y. Simmons, and D. A. Ritchie, Phys. Rev. B 60, 4850 (1999); J. Cunningham, V. I. Talyanskii, J. M. Shilton, M. Pepper, A. Kristensen, and P. E. Lindelof, Phys. Rev. B 62, 1564 (2000); V. I. Talyanskii, D. S. Novikov, B. D. Simons, and L. S. Levitov, Phys. Rev. Lett. 87, 276802 (2001); P. J. Leek, M. R. Buitelaar, V. I. Talyanskii, C. G. Smith, D. Anderson, G. A. C. Jones, J. Wei, and D. H. Cobden, Phys. Rev. Lett. 95, 256802 (2005).

[21] O. Entin-Wohlman, A. Aharony and Y. Levinson, Phys. Rev. B 65, 195411 (2002).

[22] A. Dhar and B. S. Shastry, Phys. Rev. B 67, 195405 (2003).

[23] A. Dhar and D. Sen, Phys. Rev. B 73, 085119 (2006).

[24] N. Bushong, N. Sai, and M. Di Ventra, Nano Lett. 5, 2569 (2005).

[25] S. Datta, Electronic transport in mesoscopic systems (Cambridge University Press, 1995); Y. Meir and N. S. Wingreen, Phys. Rev. Lett. 68, 2512 (1992).

[26] D. E. Feldman and Y. Gefen, Phys. Rev. B 67, 115337 (2003).

[27] P. Sharma and C. Chamon, Phys. Rev. B 68, 035321 (2003).

[28] D. Makogon, V. Juricic, C. Morais Smith, Phys. Rev. B 74, 165334 (2006).

[29] S. Das and S. Rao, Phys. Rev. B 71, 165333 (2005). 